\title{
Yukawa couplings and proton decay in SUSY models
}

\author{
Mario E. Gómez \\ Physics Division, School of Technology, \\ Aristotle University of Thessaloniki, \\ Thessaloniki, GR-540 06, Greece \\ E-mail: imgomez@cc.uoi.gn
}

ABstract: We discuss proton decay induced by dimension- 5 operators in supersymmetric models containing extra hypercharge $-1 / 3$ colour-triplets. We derive a general formula relating dimension -5 operator to the colour-triplet mass matrix. We show that certain zeros in the triplet mass-matrix together with some triplet coupling selection rules can lead to elimination of dimension-5 operators. We apply this mechanism to $S U(5)$ and flipped $S U(5)$ theories with extended Higgs sectors.

\section{Introduction}

Proton decay is a generic feature of any unification scheme since the unification of quarks and leptons in a common multiplet introduces extra interactions that violate baryon number. Proton decay rates and modes are a prediction of GUT models that play a crucial role in their phenomenological viability. In fact, proton decay has turned out to be the nemesis of many GUT and Superstring models. It is a welcome prediction that can be used to test GUTs. In supersymmetric GUTs with conserved $R$-parity the dominant baryon number violating operators are dimension $D=5$, while $D=6$ operators are in general suppressed due to the increase of the unification scale in comparison to its nonsupersymmetric values. $D=5$ operators are proportional to the Yukawa couplings and to the inverse of the heavy mass [1]. In minimal models the Yukawa couplings involved are associated with the fermion masses. The values of these couplings play an important role in the final value of the proton decay rate and the resulting hierarchy of existing modes. Nevertheless, Superstring embeddable models [2] or models of phenomenologically oriented GUTs that treat the fermion mass problem [패] Higgs sector.

In this talk we summarize the results of a recent work [īi], where we propose a mechanism for eliminating or suppressing such operators based on the use of textures of the hypercharge $1 / 3$ mass-matrix accompanied by certain constraints of the extra triplet coupling to matter.

\section{Proton Decay in minimal SU(5) models}

Let us consider unified models with the minimal Higgs content to allow the beaking of the $S U(5)$ symmetry to $S U(3)_{c} \times S U(2)_{L} \times U(1)_{Y}$ at the GUT scale $M_{G U T}$.

Non Supersymmetric $S U(5)$ models predict proton decay as a consequence of gauge interactions of the heavy particles, originated when the $S U(5)$ symmetry is broken, and quarks and leptons. The baryon-number-vioalting operators are $D=6$ and they are suppressed as the square of the mass of the heavy particles $\left(\approx M_{G U T}\right)$. The dominant proton decay mode in these models is $p \rightarrow e^{+} \pi^{0}$. The calculated lifetime [in :

$$
\tau\left(p \rightarrow e^{+} \pi^{0}\right) \approx\left(\frac{M_{G U T}}{3.5 \cdot 10^{4} \mathrm{GeV}}\right)^{4} \times 10^{31 \pm 1} y r
$$

While the experimental bound for this process is [i $[\overline{6}]$

$$
\tau\left(p \rightarrow e^{+} \pi^{0}\right)>5.5 \times 10^{32} \text { years }
$$

Since tipical values for the cuasi unification in non-susy $S U(3)_{c} \times S U(2)_{L} \times U(1)_{Y}$ are $M_{G U T} \approx$ 
$10^{13}-10^{14}$ Gev, proton lifetime predictions exceeds the experimental bounds.

In SUSY SU(5) models, the scale of unification is increased to $M_{G U T} \approx 10^{16} \mathrm{GeV}$, this is enought to bring the prediction for $D=6$ mediated proton decay to a safe limit:

$$
\tau\left(p \rightarrow e^{+} \pi^{0}\right) \approx 10^{35 \pm 1} \text { years }
$$

However proton decay is predicted, at smaller rates, due to Yukawa interactions. In this case the supersymmetric partners of the colored triplet Higgs bosons interact with leptons (sleptons) and quarks (squarks) fields. $D=5$ operators arises suppresed only by one power of $M_{G U T}$.

Color triplets are contained in Higgs pentaplets $h, \bar{h}$. The quarks and leptons are assigned to $\phi(\overline{\mathbf{5}})+\psi(\overline{\mathbf{1 0}})$ representations of $S U(5)$. The part of the superpotential related to dimension -5 decay will be

$$
Y_{i j}^{u} \psi_{i} \psi_{j} h_{1}+Y_{i j}^{d} \psi_{i} \phi_{j} \bar{h}_{1}+\mu h \bar{h}+\lambda h \Sigma \bar{h}
$$

where the symbol $\Sigma$ stands for the adjoint Higgs superfield in the $\mathbf{2 4}$ representation.

The $S U(5)$ symmetry is broken down to the MSSM, when $\Sigma$ gets a VEV, V, along the 24direction. The isodoublet and colour-triplet masses are

$$
\begin{gathered}
M_{2}=\mu-3 \lambda V, \\
M_{3}=\mu+2 \lambda V
\end{gathered}
$$

The triplets are heavy, $M_{3} \sim M_{G U T}$, while the doublet pair must remain light $M_{2} \sim m_{w}$. Hence a fine-tuning condition must be imposed in the parameters of the superpotential (2.2i).

The effective $S U(3)_{c} \times S U(2)_{L} \times U(1)_{Y}$ superpotential describing the couplings of quarks and leptons to the extra coloured triplets of the $D$-quark type

$$
Y_{i j}^{u} Q_{i} Q_{j} D+Y_{i j}^{d} Q_{i} L_{j} \bar{D}+Y_{i j}^{u} E_{i}^{c} U_{j}^{c} D,
$$

$D=5$ operators can be converted into fourfermion operators by the appropiate gaugino dressing. Assuming roughly an overall universal supersymmetry breaking scale $m_{S}$, the corresponding four-fermion operator will involve:

$$
\lambda \cdot\left[\frac{\left(M_{3}\right)^{-1}}{m_{S}}-4 m_{S}\left(M_{3}\right)^{-3} \log \frac{\left(M_{3}\right)}{m_{S}}\right]
$$

Where $\lambda$ contain a combination of Yukawa and gauge couplings.

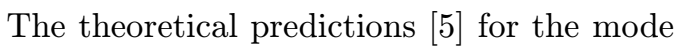
$p \rightarrow \bar{\nu} K$ are comparable to the experimental

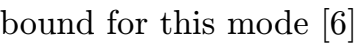

$$
\tau(p \rightarrow \bar{\nu} K)>5.5 \times 10^{32} y r .
$$

Hence, the parameter space for the minimal SUSY-SU(5) is very restricted.

In $S U(5)$-models with a non-minimal content of Higgs multiplets, $M_{3}^{-1}$ of eq. (2.6) will be replaced by a matrix, and therefore its null elements will play an important role in the suppresion of $D=5$-operator mediated proton decay.

In the minimal flipped $S U(5) \times U(1)$ model [i] $\left.\bar{i}_{1}\right]$ matter fields come in the representations

$$
F_{i}(\mathbf{1 0}, 1 / 2), \quad f_{i}^{c}(\overline{\mathbf{5}},-3 / 2), \quad l_{i}^{c}(\mathbf{1}, 5 / 2)
$$

while Higgses in

$$
h(\mathbf{5},-1), \bar{h}(\overline{\mathbf{5}}, 1),
$$

and in

$$
F_{h}(\mathbf{1 0}, 1 / 2), \quad \bar{F}_{h}(\overline{\mathbf{1 0}},-1 / 2) .
$$

The part of the superpotential relevant for the beaking of the unifiying symmetry and Yukawa terms is:

$$
\begin{aligned}
& f_{i j} F_{i} F_{j} h+y_{i j} F_{i} f_{j}^{c} \bar{h}+r_{i j} l_{i}^{c} f_{j}^{c} \bar{h}+ \\
& \mu_{h} \bar{h}+\lambda F_{h} F_{h} h+\overline{\lambda F}_{h} \bar{F}_{h} \bar{h}
\end{aligned}
$$

VEV's of $F_{h}$ and $\overline{F_{h}}$ along the neutrino-like component break the $S U(5) \times U(1)$ symmetry to the MSSM. A great advantage of the "flipped" $S U(5)$ model over the ordinary one is that of the realization of the "triplet-doublet splitting" mechanism without fine-tuning the parameters of the superpotential ( blet mass is given by the parameter $\mu$ which can be $\sim m_{w}$ while the mass matices for the triplets:

$$
\mathcal{M}_{3}=\left(\begin{array}{cc}
0 & \lambda V \\
\overline{\lambda V} & 0
\end{array}\right)
$$

Where the entry 22 is null since the pair $F_{h} \bar{F}_{h}$ has to be massless in order to realize the $S U(5) \times$ $U(1)$ breaking to the standard model. Since in this model there is not $D \bar{D}$ mass term, $D=5$ operators are naturally suppresed. 


\section{How to suppress dimension-5 op- erators in effective models with extra triplets}

Let us consider a general supersymmetric model containing some extra hypercharge $-1 / 3$ colourtriplets ${ }^{1}$. The effective $S U(3)_{c} \times S U(2)_{L} \times U(1)_{Y}$ superpotential describing the couplings of quarks and leptons to the extra coloured triplets of the $D$-quark type

$$
f_{i j}^{\alpha} Q_{i} Q_{j} D_{\alpha}+y_{i j}^{\alpha} Q_{i} L_{j} \bar{D}_{\alpha}+r_{i j}^{\alpha} E_{i}^{c} U_{j}^{c} \bar{D}_{\alpha}
$$

where $i, j=1,2,3$ are the usual generation indices and $\alpha=1, \ldots, N$ is an extra index describing the multiplicity of triplets and repeated indices are summed. In addition the effective triplet mass matrix will be of the form

$$
\left(\mathcal{M}_{3}\right)_{\alpha \beta} D_{\alpha} \bar{D}_{\beta}
$$

where $\mathcal{M}_{3}$ is in general non-diagonal.

We can always go to a basis in which the triplet mass-matrix is diagonal

$$
\begin{gathered}
D_{\alpha}=S_{\alpha \beta} D_{\beta}^{\prime}, \bar{D}_{\alpha}=U_{\alpha \beta} \bar{D}_{\beta}^{\prime} \\
\mathcal{M}_{3 D} \equiv \operatorname{diag}\left(m_{1}, m_{2}, \cdots, m_{N}\right)=S^{T} \mathcal{M}_{3} U
\end{gathered}
$$

where the matrices $S$ and $U$ are unitary. In this basis we can easily evaluate $D=5$ operators resulting from Higgs triplet fermion exchange, and then recast the result in the original basis. Assuming that all triplets are massive $\left(m_{i} \neq\right.$ $0, i=1, \ldots, N)$, operators with the structure $Q_{i} Q_{j} Q_{k} L_{n}$ will be proportional to ${ }^{2}$

$$
\begin{aligned}
\mathcal{O}_{i j k l}^{Q Q Q L} & =\sum_{\alpha, \beta, \gamma=1}^{N} f_{i j}^{\alpha} S_{\alpha \gamma}\left(\mathcal{M}_{3}{ }_{D}^{-1}\right)_{\gamma} U_{\beta \gamma} y_{k n}^{\beta} \\
& =\sum_{\alpha, \beta=1}^{N} f_{i j}^{\alpha}\left(\mathcal{M}_{3}{ }^{-1}\right)_{\alpha \beta}^{T} y_{k n}^{\beta} \\
& \left.=\frac{1}{\operatorname{det}\left(\mathcal{M}_{3}\right)} \sum_{\alpha, \beta=1}^{N} f_{i j}^{\alpha} \operatorname{cof}\left(\mathcal{M}_{3}\right)_{\alpha \beta} y_{k n}^{\beta} .5\right)
\end{aligned}
$$

\footnotetext{
${ }^{1}$ This superpotential arises in the case of the standard $S U(5)$ with extra Higgs 5-plets or from the flipped $S U(5) \times U(1)$ with both extra Higgs 5 -plets and 10-plets.

${ }^{2}$ The corresponding four-fermion operator, assuming roughly an overall universal supersymmetry breaking scale $m_{S}$, will involve $\frac{\left(\mathcal{M}_{3}\right)^{-1}}{m_{S}}-4 m_{S}\left(\mathcal{M}_{3}\right)^{-3} \log \frac{\left(\mathcal{M}_{3}\right)}{m_{S}}$.
}

Analogous formulas hold for $D=5$ operators of the type $Q_{i} Q_{j} U_{k}^{c} E_{k}^{c}$.

Suppose now that we want to eliminate all dimension five operators. Assuming that the Yukawa couplings $f_{i j}^{\alpha}$ and $y_{i j}^{\beta}$ are in general unrelated and $\operatorname{det} \mathcal{M}_{3} \neq 0$, equation (3.5i) implies that the necessary and sufficient condition for vanishing of the $\mathcal{O}_{i j k l}^{Q Q Q L}$ operator is that for every pair of triplets $\left(D^{\alpha}, \bar{D}^{\beta}, \alpha, \beta=1, \ldots, N\right)$ that $d o$ couple to quarks and leptons respectively $\left(f_{i j}^{\alpha} \neq\right.$ 0 and $\left.h_{i j}^{\beta} \neq 0\right)$ the cofactor of the corresponding triplet mass matrix element $\left(\mathcal{M}_{3}\right)_{\alpha \beta}$ vanishes ${ }^{3}$

$$
\begin{aligned}
& \mathcal{O}_{i j k l}^{Q Q Q L}=0 \Longleftrightarrow \operatorname{cof}\left(\mathcal{M}_{3}\right)_{\alpha \beta}=0 \\
& \forall(\alpha, \beta) \in \Xi=\left\{(\alpha, \beta): f_{i j}^{\alpha} \neq 0\right. \\
& \text { and } \left.h_{k l}^{\beta} \neq 0\right\} .
\end{aligned}
$$

It is obvious that in the case where all triplets ( $D$ 's and $\bar{D}$ 's) couple to matter the suppression of dimension five operators (3.5i) is not possible since (13.6) leads to $\operatorname{det}\left(M_{3}\right)=0$. Nevertheless, if for some reason (discrete symmetry, $\mathrm{R}$-parity, anomalous $U(1)$, accidental symmetry) some of the $f_{i j}^{\alpha}$ and/or $y_{k l}^{\beta}$ are zero and the triplet mass matrix is such that the cofactors of the appropriate matrix elements are zero then the associated dimension-5 operator vanishes.

The previous discussion leads to the possibility that in a model with extra $D$-quark triplets dimension-5 operators can be eliminated by using textures of triplet mass matrices and the tripletmatter couplings.

To be concrete let us give a simple example of such an effective theory. Consider the case of an effective theory with two extra triplets. Only the first couples to matter through the superpotential terms

$$
f_{i j}^{1} Q_{i} Q_{j} D_{1}+y_{i j}^{1} Q_{i} L_{j} \bar{D}_{1}+r_{i j}^{1} E_{i}^{c} U_{j}^{c} \bar{D}_{1}
$$

and their mass-matrix has the form

$$
\mathcal{M}_{3}=\left(\begin{array}{cc}
\mu_{11} & \mu_{12} \\
\mu_{21} & 0
\end{array}\right) \text {. }
$$

Since $f_{i j}^{2}=y_{i j}^{2}=0$ evaluation of (3.5i) leads to

$$
\mathcal{O}_{i j k l}^{Q Q Q L}=f_{i j}^{1} \operatorname{cof}\left(\mathcal{M}_{3}\right)_{11} y_{k n}^{1} \sim \operatorname{cof}\left(\mathcal{M}_{3}\right)_{11}=0
$$

\footnotetext{
${ }^{3}$ We consider here the triplet $D^{\alpha}$ as coupled to quarks and leptons if at least one $f_{i j}^{\alpha} \neq 0$ and similarly for antitriplets.
} 
It is remarkable that if we remove the second pair of triplets of the model (which do not couple directly to matter) the usual dimension-5 operators reappear. We shall study below that this nice property can be incorporated in $S U(5)$ models.

\section{4. $S U(5)$ models without dimension- 5 operators}

Let us consider now an $S U(5)$ model with two pairs of Higgs pentaplets $h_{\alpha}, \bar{h}_{\alpha}, \alpha=1,2$ of which only the first couples to matter. The quarks and leptons are assigned to $\phi(\overline{\mathbf{5}})+\psi(\overline{\mathbf{1 0}})$ representations of $S U(5)$. The part of the superpotential related to dimension -5 decay will be

$$
\begin{aligned}
& f_{i j} \psi_{i} \psi_{j} h_{1}+y_{i j} \psi_{i} \phi_{j} \bar{h}_{1}+ \\
& \sum_{\alpha, \beta=1}^{2}\left(\mu_{\alpha \beta} h_{\alpha} \bar{h}_{\beta}+\lambda_{\alpha \beta} h_{\alpha} \Sigma \bar{h}_{\beta}\right)
\end{aligned}
$$

where the symbol $\Sigma$ stands for the adjoint Higgs superfield in the $\mathbf{2 4}$ representation. The isodoublet and colour-triplet mass matrices are correspondingly of the form

$$
\begin{gathered}
\mathcal{M}_{2}=\mu-3 \lambda V, \\
\mathcal{M}_{3}=\mu+2 \lambda V
\end{gathered}
$$

The well known fine-tuning that guarantees a massless pair of isodoublets amounts to

$$
\operatorname{det}\left(\mathcal{M}_{2}\right)=0
$$

The proton decay rate through $D=5$ operators is, according to equation (3.5i), determined by the cofactor of the $1-1$ element of the triplet mass matrix

$$
\operatorname{cof}\left(\mathcal{M}_{3}\right)_{11}=\left(\mu_{22}+2 \lambda_{22} V\right)
$$

Hence, choosing $\mu_{22}=-2 \lambda_{22} V$ dimension-5 operators vanish. This condition is perfectly compatible with the previous fine-tuning condition $(4.4)$. It is very interesting that proton decay through $D=5$ operators can be set to zero through a condition on the couplings ${ }^{4}$.

\footnotetext{
${ }^{4}$ Of course, proton decay still goes through at the (suppressed) rate of $D=6$ operators.
}

In the framework of our standard $S U(5)$ example the required zero in the inverse triplet mass matrix does not correspond to any symmetry and is in a sense a second fine-tuning. Nevertheless, the general conclusion is that zeros of the triplet mass matrix, perhaps attributable to symmetries, can stabilize the proton.

The superpotential considered above in (4.1.1) is not the most general one. In fact, the case that all 5-plets couple to matter cannot be reduced to $\left(\mathbf{A}^{\prime} . \overline{1_{1}^{\prime}}\right)$ since it would require a different Higgs 5-plet rotation for each generation of matter. However, we should emphasize the fact that in $S U(5)$ models with non minimal Higgs content, the constraints imposed by proton decay on the parameter space and triplet masses can be relaxed.

\section{Dimension-5 operators in exten- sions of the flipped $S U(5)$}

In spite of the nice features of the minimal flipped $S U(5)$ model, all attempts to obtain such a model from strings have yielded up to now non-minimal models. Such models include

(a) extra pairs of low energy Higges $(h, \bar{h})$ and/or (b) extra pairs of $S U(5) \times U(1)$ breaking Higges $\left(F_{h}, \bar{F}_{h}\right)$.

We are thus motivated to study the presence of dimension- 5 operators in such models. As we shall see contrary to the minimal case, such extensions of the flipped $S U(5)$ model are not automatically free of dimension -5 operators.

The relevant part of the superpotential assuming $N_{5}$ pairs of Higgs 5 -plets $\left(h_{\alpha}, \bar{h}_{\alpha}, \alpha=\right.$ $\left.1, \ldots, N_{5}\right)$ that couple to matter and $N_{10}$ pairs of Higgs $10-$ plets $\left(F_{h \alpha}, \bar{F}_{h A}, A=1, \ldots, N_{10}\right)$ that do not couple to matter, will have the form

$$
\begin{aligned}
& f_{i j}^{\alpha} F_{i} F_{j} h_{\alpha}+y_{i j}^{\alpha} F_{i} f_{j}^{c} \bar{h}_{\alpha}+ \\
& r_{i j}^{\alpha} l_{i}^{c} f_{j}^{c} \bar{h}_{\alpha}+\mu_{\alpha \beta} h_{\alpha} \bar{h}_{\beta}+m_{A B} F_{h A} \bar{F}_{h B} \\
& +\lambda_{A B \gamma} F_{h A} F_{h B} h_{\gamma}+\bar{\lambda}_{A B \gamma} \bar{F}_{h A} \bar{F}_{h B} \bar{h}_{\gamma}(5.1)
\end{aligned}
$$

where $A, B=1, \cdots, N_{10}, \alpha, \beta, \gamma=1, \cdots, N_{5}$. Assuming GUT symmetry breaking to an arbitrary direction in the Higgs 10-plet space $\left(\left(V_{1}, V_{2}, \ldots, V_{N_{10}}\right) \text { and similarly for bars }\right)^{5}$, we

\footnotetext{
${ }^{5}$ D-flatness requires $\sum_{A} V_{A}^{2}=\sum_{A} \bar{V}_{A}{ }^{2}$
} 
obtain the triplet mass matrix ${ }^{6}$

$$
\mathcal{M}_{3}=\left(\begin{array}{cc}
\mu_{\alpha \beta} & v_{\alpha A} \\
\bar{v}_{A \beta} & m_{A B}
\end{array}\right)
$$

where $\mu_{\alpha \beta}$ is the doublet mass-matrix and $v_{\alpha A}=$ $2 \lambda_{A B \alpha} V_{B}, \bar{v}_{A \beta}=2 \bar{\lambda}_{A B \beta} \bar{V}_{B}$

$\mathrm{F}$-flatness demands $\operatorname{det}(m)=0$ in order to have at least one pair of massless Higgs decuplets which will realize the GUT symmetry breaking. One can actually choose $m$ to have only one zero eigenvalue so that all remnants of the Higgs decuplets will become heavy.

Let us now start our study by a simple example. Consider the flipped model with two pairs of Higgs 5-plets and one pair of Higgs 10-plets. Assuming for simplicity that the 5 -plet mass matrix is diagonal, the explicit form of the triplet matrix is 7

$$
\mathcal{M}_{3}=\left(\begin{array}{ccc}
0 & 0 & \lambda_{1} V \\
0 & \mu & \lambda_{2} V \\
\bar{\lambda}_{1} \bar{V} & \bar{\lambda}_{2} \bar{V} & 0
\end{array}\right)
$$

and $\operatorname{det}\left(\mathcal{M}_{3}\right)=\lambda_{1} \bar{\lambda}_{1} \bar{V}$ The transpose of inverse triplet matrix entering in formula (3.5i) is

$$
\left(\mathcal{M}_{3}^{-1}\right)^{T}=\left(\begin{array}{ccc}
\frac{\lambda_{2} \bar{\lambda}_{2}}{\lambda_{1} \bar{\lambda}_{1} \mu} & -\frac{\lambda_{2}}{\lambda_{1} \mu} & \cdot \\
-\frac{\bar{\lambda}_{2}}{\bar{\lambda}_{1} \mu} & \frac{1}{\mu} & \cdot \\
\cdot & \cdot & \cdot
\end{array}\right)
$$

where the dots stand for elements which are irrelevant. It is now obvious that in this model dimension five operators cannot be eliminated since even in the case $\bar{\lambda}_{2}=\lambda_{2}=0$ the 22 element does not vanish. If we want to eliminate them we have two solutions :

(i) assume that the extra pair of 5-plets does not couple to matter. In this case only the 11 element of the matrix in (15.4) is relevant and it vanishes for $\lambda_{2}=0$ (or $\bar{\lambda}_{2}=0$ ).

(ii) make the milder assumption that one of the 5-plets (e.g. $h_{2}$ ) does not couple to the up quarks (or similarly $\bar{h}_{2}$ does not couple to the down). In this case the second column (or line) of the matrix in $(5.4)$ becomes irrelevant and the column (or line) left vanishes for $\lambda_{2}=0$ (or $\bar{\lambda}_{2}=0$ ).

\footnotetext{
${ }^{6}$ In a $\left(D_{1}, \cdots, D_{N_{5}},\left(d_{H}^{c}\right)_{1}, \cdots,\left(d_{H}^{c}\right)_{N_{10}}\right) \quad$ versus $\left(\bar{D}_{1}, \cdots, \bar{D}_{N_{5}},\left(\bar{d}_{H}^{c}\right)_{1}, \cdots,\left(\bar{d}_{H}^{c}\right)_{N_{10}}\right)$ basis, where with $D$ we denote the triplets which lie inside the Higgs 5-plets and with $D_{H}$ the triplets that lie inside the Higgs 10-plets.

${ }^{7}$ We have renamed $\lambda_{1}=\lambda_{111}, \lambda_{2}=\lambda_{112}$.
}

Another case that could arise is the existence of extra decuplets. The simplest of these cases is for $N_{5}=1$ and $N_{10}=n \geq 2$.

$$
\operatorname{cof}\left(\mathcal{M}_{3}\right)_{11}=\frac{\operatorname{det} m}{\operatorname{det} \mathcal{M}_{3}}
$$

which means that proton decay is absent only in the case that the restricted mass-matrix of the triplets not coupled to matter has

$$
\operatorname{det} m=0
$$

This constrain naturally arises in the context of the flipped $S U(5) \times U(1)$ model as consequence of $\mathrm{F}$-flatness as we mentoned above.

In the more general case where $N_{5}$ and $N_{10}$ are arbitrary dimension -5 operators can be suppressed only in the case $N_{5} \leq N_{10}$. Furthermore one has to require that the Higgs decuplet mass matrix has $N_{5}$ zero eigenvalues. This is compatible with symmetry breaking and with the requirement of making all triplets heavy but leaves $N_{5}-1$ pairs of $Q(\mathbf{3}, \mathbf{2}, 1 / 6)+\bar{Q}(\overline{\mathbf{3}}, \mathbf{2},-1 / 6)$ massless. This feature does not necessarily mean that this possibility is ruled out. On the contrary one can consider the cases where extra $Q$ 's have intermediate masses which are small enough to sufficiently suppress dimension -5 operators but they are still compatible with renormalization group requirements. The appearance of extra vectorlike pairs of $Q$ and $D$ type multiplets with intermediate masses is a welcomed feature in the context of flipped $S U(5) \times U(1)$ models that raise the unification scale to the string scale [īind.

\section{Conclusions}

Our main result is that textured zeros of the color-triplet mass-matrix as well as Yukawa selection rules can eliminate certain dimension- 5 operators. In order to be specific we focused on $S U(5)$ models. In particular, we showed that introducing an extra pair of Higgs pentaplets in the standard supersymmetric $S U(5)$, with specific couplings, can eliminate these operators. We also considered the case of the flipped-SU(5) model with extra pentaplets and decuplets and analyzed the conditions for vanishing proton decay through dimension- 5 operators. Flipped- $S U(5)$ with extra decuplets was shown to be $D=5$ 
operator-free as it happens in the case of the minimal model. However, flipped-SU(5) with extra Higgs pentaplets is not automatically free of dimension -5 operators. We have proposed a solution to this problem which involves a texture of the pentaplet matrix together with certain constraints on the pentaplet couplings to matter.

\section{Acknowledgments}

This work is supported by E.U. under the TMR contract "Beyond the Standard Model", ERBFMRX-CT96-0090.

\section{References}

[1] S. Weinberg, Phys. Rev. D26 (1982) 287; N. Sakai and T. Yanakida, Nucl. Phys. B197 (1982) 533.

[2] see e.g.

I. Antoniadis and G. Leontaris, Phys. Lett. B216 (1989) 333;

S. Ranfone and J. W. F. Valle, Phys. Lett. B386 (1996) 151;

J. Rizos and K. Tamvakis, Phys. Lett. B414 (1997) 277.

[3] see e.g. K. S. Babu and S. M. Barr, Phys. Rev. Lett. 75 (1995) 2088.

[4] M. E. Gómez, J. Rizos and K. Tamvakis Phys. Rev. D59 (1998)015015.

[5] P. Nath and R. Arnowitt, hep-ph/9808465 and references therein.

[6] C. Caso it et al., The European Physics Journal C3(1998)1.

[7] S. M. Barr, Phys. Lett. B112 (1982) 219;

J. P. Derendinger, J. H. Kim, D. V. Nanopoulos, Phys. Lett. B194 (1987) 231.

[8] J. L. Lopez and D. V. Nanopoulos, Nucl. Phys. B399 (654) 1993;

see also J. L. Lopez, D. V. Nanopoulos and A. Zichichi, hep-ph 9307211 and references therein. 